\title{
Mechanisms of immune privilege in the anterior segment of the eye: what we learn from corneal transplantation
}

\author{
Junko Hori
}

Received: 12 February 2008 / Accepted: 16 July 2008 / Published online: 8 August 2008

(C) Humana Press 2008

\begin{abstract}
The eye, like the brain and reproductive organs, possesses inherent immune privilege, and inflammation is self-regulated so as to preserve the organ functions. Studies over the past 30 years have provided insights of the multiple mechanisms of immune privilege. At present, three major lines of thought prevail regarding the molecular mechanisms of immune privilege in the eye: there are (1) anatomical, cellular, and molecular barriers in the eye; (2) eye-derived immunological tolerance, the so-called anterior chamber-associated immune deviation; and (3) immune suppressive intraocular microenvironment. In this review, the mechanisms of immune privilege that have been learned from ocular inflammation animal models, especially corneal transplantation, are described. Roles of new B7 family molecules on local immune regulation within the cornea are also introduced.
\end{abstract}

Keywords Immune privilege · Immune suppression · Corneal transplantation · ACAID · Costimulatory molecules

The eye is constructed from tissue with little or no capacity for regeneration, specifically, corneal endothelial cells and retinal cells cannot proliferate in vivo. For that reason, ocular tissue damage due to excessive inflammation can lead to loss of sight. Accordingly, the eye, like the brain and reproductive organs, is endowed with immune privilege [1,

\section{J. Hori $(\square)$}

Department of Ophthalmology, Nippon Medical School,

Sendagi 1-1-5, Bunkyo-ku,

Tokyo 113-8603, Japan

e-mail: jhori-tky@umin.ac.jp
2], and inflammation is self-regulated so as to preserve the organ functions. Animal studies have shown that when eyes are deprived of immune privilege, autoimmune intraocular inflammation readily occurs, and that condition is severe and intractable [1, 2]. In addition, corneal transplants are the least-rejected among all organ transplants, and that characteristic is also attributable to immune privilege $[1,2]$. When corneal transplantation is performed on eyes that have been deprived of immune privilege, the rejection rate becomes as high as in the cases of heart and skin transplantation [1-3].

The concept of immune privilege was first proposed in the 1940s by the Nobel laureate, P.B. Medawar, and colleagues [4]. They recognized the extended survival of foreign tissue grafts placed in the anterior chamber of the eye or in the brain. At that time, it was thought that the eyes and brain had an anatomical barrier and that the lesser rejection of transplants was due to immunological ignorance [4]. However, more recently, it has been elucidated by Streilein and colleagues, that it is not just due to immunological ignorance, but also an active immune suppression mechanism [1, 2]. Progress has been made in analyzing the regulatory mechanisms in ocular inflammation by using animal models of corneal transplantation and autoimmune uveitis. At present, three major lines of thought prevail regarding the molecular mechanisms of immune privilege in the eye: (1) there are anatomical, cellular, and molecular barriers in the eye; (2) eye-derived immunological tolerance, the so-called anterior chamberassociated immune deviation (ACAID); and (3) immune suppressive microenvironment in the eye. Here, I review the mechanisms of immune privilege in the eye by using a corneal transplantation model and also describe role of new B7 family molecules on local immune regulation in the anterior segment. 


\section{Anatomical and cellular barriers in the cornea}

The cornea contains no blood or lymph vessels; therefore, considerable time passes after corneal transplantation before antigen recognition occurs in the regional lymph nodes and effector cells reach the graft. In addition, the corneal epithelial cells, keratocytes, and endothelial cells, do not express MHC class II molecules and also express only low levels of MHC class I molecules [5,6]. This means that the main targets of a rejection reaction are not $\mathrm{MHC}$ antigens, but the minor $\mathrm{H}$ antigens in the corneal allografts [7]. When corneal endothelial cells were simultaneously exposed to interferon (IFN)- $\gamma$ and tumor necrosis factor (TNF)- $\alpha$, they expressed MHC class II molecules but not the class II transactivator gene or invariant chain [8]. In addition, MHC class II-positive dendritic cells and macrophages are absent in normal cornea, except in the peripheral region of the cornea [5]. There are small numbers of bone marrow-derived cells that still do not express MHC class II antigens in normal cornea, but controversy remains over whether they play the role of mature antigen-presenting cells (APCs), and it is thought that there are no donorderived mature APCs in the donor cornea [9]. Therefore, the role of antigen presentation in the regional lymph nodes after corneal transplantation is carried out mainly by hostderived APCs rather than donor-derived APCs, and it is thought that recognition of donor antigens occurs in indirect fashion, by host $\mathrm{CD}^{+} \mathrm{T}$ cells via donor antigen-bearing host MHC class II molecules on host APCs [7, 10]. The main effector mechanism of rejection reactions in corneal transplantation is thought to be delayed-type hypersensitivity (DTH), rather than cytotoxic T cells (CTL), with the reaction targeting the donor minor $\mathrm{H}$ antigens rather than the donor MHC molecules [7, 10]. Thus, it can be surmised that the post-transplantation immune response is weaker in the case of corneal transplants compared with other organ grafts because of not only the anatomical characteristics of the corneal tissue but also its low antigenicity and the above-described mechanisms of antigen presentation and recognition.

\section{Anterior chamber-associated immune deviation}

Anterior chamber-associated immune deviation refers to a phenomenon in which antigen-specific systemic immunological tolerance is induced to an antigen that has been introduced to the anterior chamber in rodent eyes [1, 2]. ACAID is a phenomenon in which antibody responses are preserved while cellular responses such as DTH and CTL are suppressed. It is known that similar phenomenon occurs even in relation to an antigen that has been introduced the vitreous cavity or the subretinal space of the eye [1]. ACAID is induced in relation to various kinds of antigens, including allo-transplantation antigens, soluble protein antigens, viral antigens, and tumor antigens, and it has been demonstrated to be involved in various events such as acceptance of corneal transplants, autoimmune uveitis, acute retinal necrosis (ARN) in a fellow eye that experienced herpes virus infection in the anterior segment, or progression of intraocular malignant melanoma, in mouse models [1]. Induction of ACAID to autoantigens is reportedly induced by inoculating the anterior chamber with retinal autoantigens followed by a demonstration that such mice are subsequently resistant to the induction of experimental autoimmune uveitis by immunization with the same autoantigen [11]. In several areas, the findings from studies in the mouse model can be extrapolated to understanding the pathogenesis in human patients. For example, patients with ARN develop an ACAID-like response to viral antigens in the intraocular compartment that disappears as the disease resolves [12-14].

The eye and the spleen are involved in the induction of ACAID (Fig. 1). Transforming growth factor (TGF)- $\beta 2$ and thrombospondin (TSP)-1 in the anterior chamber are involved in the induction of APC mediators of ACAID, and eye-derived APCs such as macrophages that express F4/80 molecule pass across the trabecular meshwork, enter the bloodstream, and reach the spleen [15-18]. The eyederived APCs that reach the marginal zone in the spleen produce TGF- $\beta$, macrophage inflammatory protein 2 , and CXC-chemokine ligand 2 and attract natural killer T (NKT) cells [19]. Then CD1d molecules that are expressed on the surface of the APCs bind with receptors that are expressed on the surface of the NKT cells, thereby presenting the antigens [20]. NKT cells produce TGF- $\beta$, IL-10, RANTES, CC-chemokine ligand 5, and TSP-1 [21, 22]. When marginal zone B cells are also present in this environment rich in immunomodulatory factors, clusters comprised of these three cell types form [23, 24]. When CD4+ and/or $\mathrm{CD} 8+\mathrm{T}$ cells that are attracted to those clusters recognize the antigens being presented by the eye-derived APCs and the marginal zone B cells, they differentiate into ACAIDinducing regulatory $\mathrm{T}$ cells (ACAID-Treg). At that time, Qa-1 molecule on the surface of the marginal zone B cells function as antigen-presenting molecule. CD4+ ACAIDTreg inhibit the differentiation for Th1 cells in secondary lymph tissues such as lymph nodes, while CD8+ ACAIDTreg inhibit the function of effector T cells (Th1 and Th2) in the local site $[25,26]$. It was recently reported that thymocytes and splenic $\gamma \delta \mathrm{T}$ cells are also necessary for induction of ACAID, and it can be understood that immune privilege in the eye is sustained through the cooperation of various cells from organs other than the eye itself (Fig. 1) [1, 2, 27].

Following corneal transplantation, ACAID is induced by the mechanism described above after the transplantation antigens on the endothelial surface of the cornea are taken 


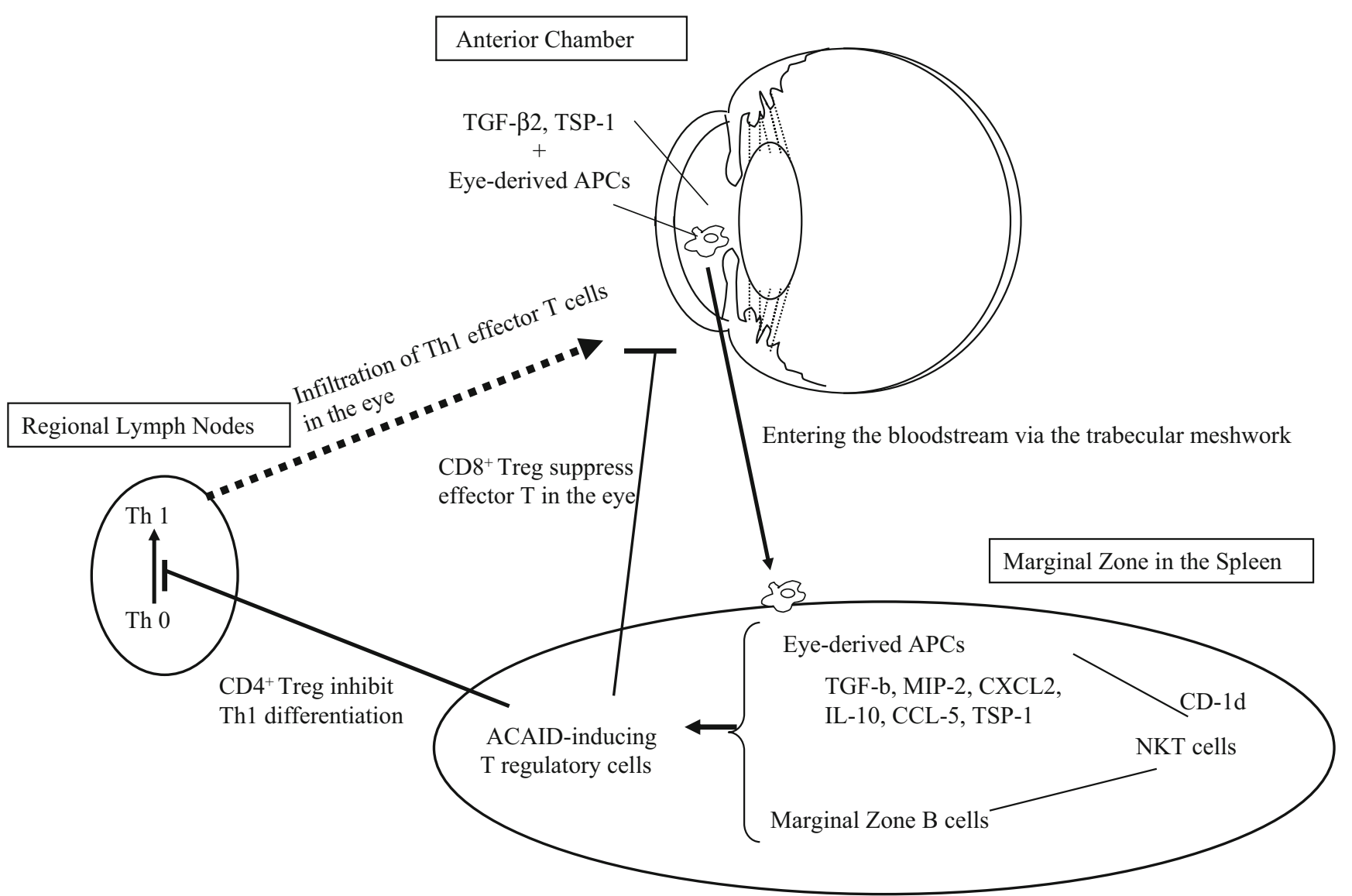

Fig. 1 Induction of ACAID. In the anterior chamber, which contains transforming growth factor (TGF)- $\beta 2$ and thrombospondin (TSP)-1, the eye-derived antigen-presenting cells have captured antigens. The eye-derived APCs enter the bloodstream, reach the marginal zone of spleen, and produce TGF- $\beta$, macrophage inflammatory protein 2 $(M I P-2)$, and CXC-chemokine ligand 2 (CXCL2). These cells attract and bind, via CD1d molecules, to NKT cells. The NKT cells produce

up by the eye-derived APCs in the anterior chamber and transported to the spleen $[1,2]$. Induction of ACAID leads to inhibition of the allo-antigen specific DTH and results in long-term survival of the graft $[10,28,29]$. ACAID cannot be induced in the case of infiltration of lymph vessels into the cornea, suturing, inflammation, trauma, or neurotomy in the cornea. In this situation, the eye is said to be at high risk of rejection, and in animal models of corneal transplantation, the rejection rate within 3 weeks after transplantation is $100 \%$ [3, 28]. Clinically, as well, rejection reactions readily occur in patients presenting this condition.

\section{Molecules maintaining an immune suppressive intraocular microenvironment}

As the anatomical and cellular barriers, or ACAID, are not absolute, innate and adaptive immune cells and molecules can still access the eye. In response to threats to vision, the
TGF- $\beta$, IL-10, CC-chemokine ligand 5 (CCL5) and TSP-1; attract marginal zone B cells; and then form clusters comprised of these three cell types. T cells, which have presented the antigens in clusters, then differentiate into ACAID-inducing regulatory $\mathrm{T}$ cells (ACAID-Treg). CD4+ ACAID-Treg inhibit the differentiation of Th1 cells in the lymph nodes, while CD8+ ACAID-Treg inhibit the function of effector Th1 and Th2 cells in the local site

eye has soluble and cell surface immunomodulatory factors that act within the oculi to suppress cells and molecules that mediate innate and adaptive immune inflammation [1]. This intraocular milieu is called the immune suppressive microenvironment. The functions of the various cells and factors that manage natural immunity and acquired immunity are inhibited by the various factors that are expressed in the anterior segment and are shown in Table $1[1,2,15$, 16, 30-39]. Among those factors, $\alpha$-melanocyte-stimulating hormone, vasoactive intestinal peptide, calcitonin generelated peptide, TGF- $\beta 2$, and TSP-1 regulate the functions of macrophages and dendritic cells. TGF- $\beta 2$ and TSP- 1 are essential factors for the induction of ACAID as described in the previous section. As shown in Table 1, various immunomodulatory factors are expressed in corneal endothelial cells and iris-ciliary body. We recently elucidated that the inhibitory costimulatory signaling molecules of the B7 family are involved in immune suppression in the cornea. Those molecules are introduced below. 
Table 1 Immunomodulatory factors expressed in the anterior segment of the eye

Soluble factors in the anterior chamber (target cells/factors to suppress)

Cell surface molecules of the cornea and iris-ciliary body

$\alpha$-MSH (T cells, macrophages, neutrophils)

B7-H1 (PD-L1) (T cells)

VIP (T cells)

Somatostatin (T cells)

CGRP (macrophages)

TGF- $\beta 2$ (T cells, macrophages, NK cells)

B7-H3 (?)

B7-2 (via CTLA4) (T cells)

TSP-1 (macrophages)

Fas L (CD95 L) (T cells, neutrophils)

MHC class Ib (T cells, NK cells)

MIF (NK cells)

IL-1Ra (IL-1)

sFas L (T cells, neutrophils)

CD46, CD55, CD59, C3ib (complement)

$\alpha$-MSH $\alpha$-Melanocyte stimulating hormone, VIP vasoactive intestinal peptide, CGRP calcitonin gene-related peptide, TGF- $\beta 2$ transforming growth factor- $\beta 2, T S P-1$ thrombospondin, $M I F$ macrophage migrating inhibitory factor, $I L-1 R a$ interleukin 1 receptor antagonist, $s F a s ~ L$ soluble Fas ligand, CTLA4 cytotoxic T lymphocyte antigen 4

\section{T-cell apoptosis mediated by B7-H1}

B7-H1 (PD-L1) was identified as a new B7 family molecule that binds to programmed death (PD)-1 on the surface of activated $\mathrm{T}$ cells and sends inhibitory signals to the $\mathrm{T}$ cells [40]. On the basis of the results generated in various animal models of autoimmune diseases, it has been reported that $\mathrm{B} 7-\mathrm{H} 1$ is involved in the induction of peripheral immune tolerance [40, 41]. In ocular tissues, B7-H1 is constitutively expressed in endothelial cells of the cornea, some stromal cells, iris-ciliary body, and the neural retina. The rejection reaction after corneal transplantation is intensified by blockade of B7-H1 or PD-1 with antibodies [39]. B7-H1 expressed in the cornea induces apoptosis of PD-1-expressing $\mathrm{T}$ cells, and deletion of effector $\mathrm{T}$ cells in the cornea results in inhibition in the effector phase of the rejection reaction [39]. It is highly interesting that the T-cell apoptosis mediated by $\mathrm{B} 7-\mathrm{H} 1$ has been only observed in immune privileged tissues or sites such as tumors, liver, and cornea so far [41, 42]. Corneal endothelial cells also constitutively express Fas ligand, and apoptosis of effector $\mathrm{T}$ cells is induced via Fas [35-37]. It is unclear whether, in this state of the effector $\mathrm{T}$ cells having been eliminated from the eye, B7-H1 and Fas ligand interact on the surface of the corneal endothelial cells (Fig. 2). On the other hand, the previously described ACAID is induced even if the PD-1/ B7-H1 pathway is blocked. That is, the induction of ACAID-Treg by the eye-derived APCs and spleen cells is not dependent on the PD-1/B7-H1 pathway.

The culture system of corneal tissue and $\mathrm{T}$ cells in vitro has been established and shown that B7-H1 expressed in the cornea shows local immunosuppressive activity [39]. This system permits complete elimination of any involvement of the secondary lymphatic organs and makes it possible to isolate and analyze only the effector phase of the rejection reaction when the corneal endothelial cells has
Fig. 2 T-cell apoptosis medicated by B7-H1 expressed in corneal endothelial cells. B7-H1, which is constitutively expressed in corneal endothelial cells, induces apoptosis of PD- $1+\mathrm{T}$ cells. Corneal endothelial cells also constitutively express Fas L, which is involved in immune regulation in the eye by inducing T-cell apoptosis via Fas

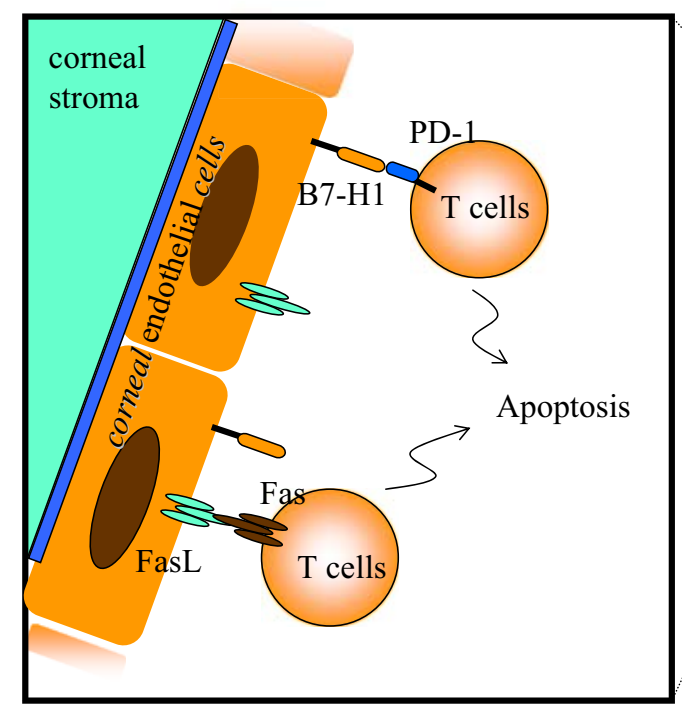


been damaged by effector $\mathrm{T}$ cells. The results showed that B7-H1 expressed in the corneal cells not only inhibited corneal endothelial damage by allo-reactive $\mathrm{T}$ cells but also inhibited bystander damage caused by activated $\mathrm{T}$ cells that are specific to third party antigen. In addition, PD-1 on the surface of the T cells was up-regulated as a result of contact with the corneal cells, thus accelerating apoptosis. Moreover, B7-H1 expression on epithelial cells was induced in the presence of inflammatory cytokine such as IFN- $\gamma$, and T-cell apoptosis mediated by B7-H1 and PD-1 was induced in each of the epithelium, stroma, and endothelium of the cornea. (Figure 3).

As described above, the PD-1/B7-H1 pathway is more involved in interactions between the effector $\mathrm{T}$ cells and the corneal cells within the eye than in the immune responses in the secondary lymphatic organs [39]. Thus, these molecules contribute to maintenance of the local immune suppressive microenvironment in the eye.

\section{ACAID induction mediated by $\mathrm{B} 7-\mathrm{H} 3$}

B7-H3 was recently identified as a new B7 family molecule, but its receptor has not yet been identified [43]. There have been conflicting reports, based on different experimental systems, as to whether B7-H3 is a costimulatory signal molecule that sends positive signals or negative signals to $\mathrm{T}$ cells [43]. This is currently a source of controversy. In the eye, B7-H3 is constitutively expressed in corneal endothelial cells and the iris-ciliary body, and that a rejection is induced after corneal transplantation in experimental hosts to which anti-B7-H3 blocking antibody had been intraperitoneally administered [44]. It is of interest that ACAID is not induced in such animals administered with anti-B7-H3 antibody. That is, B7-H3 is involved in induction of ACAID-Treg that are dependent on the spleen and mediated by eye-derived antigen-presenting cells, and it thus plays a different role from that of B7-H1 [44]. Also, the possibility that B7-H3, like the previously described $\mathrm{B} 7-\mathrm{H} 1$, plays a role in eliminating or inhibiting effector immune cells within the cornea remains to be established.

\section{Conclusion}

The eye, which is endowed with immune privilege, is a rare organ that permits analysis of the self-regulatory mechanisms for inflammation in organs. In particular, studies using animal models of corneal transplantation have revealed that a new molecule, B7-H1, induces apoptosis of effector T cells via PD-1 and plays a role in immune regulation in the cornea. In addition, we learned that another new molecule, B7-H3, is also constitutively expressed in the anterior segment of the eye and plays a role in the induction of ACAID, which is an eye-derived immunological tolerance. The normal eye possesses an anatomical barrier mechanism, ACAID, and a molecular mechanism of an immune suppressive intraocular microenvironment. It can be anticipated that understanding those
Corneal allografts

epithelium

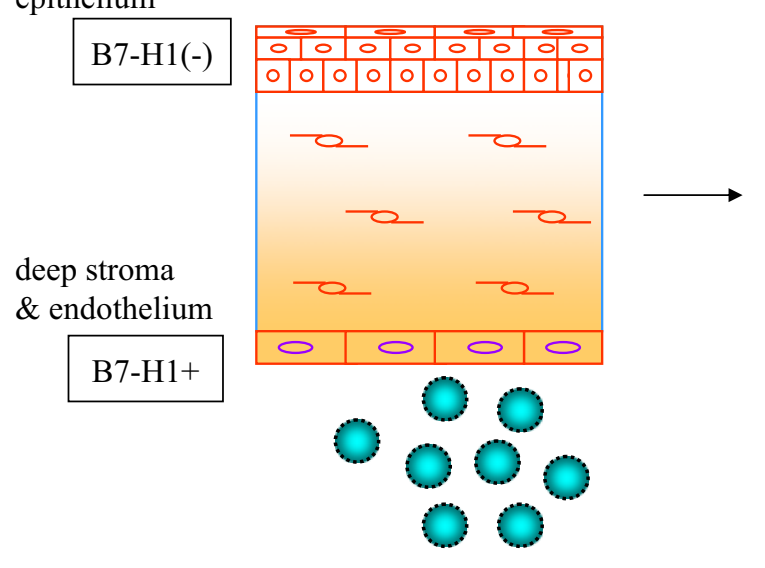

PD-1+ T cells
Induction of B7-H1 expression

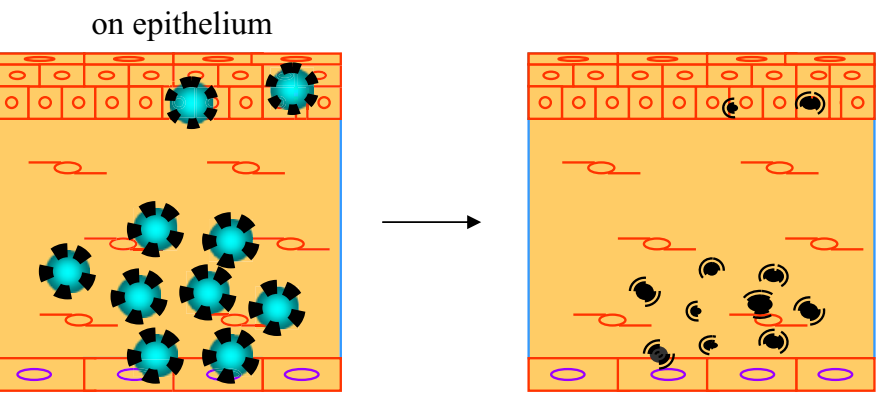

Up-regulation of

PD-1 expression on T cells
Induction of $\mathrm{T}$ cells-apoptosis in all layers of the cornea
Fig. 3 Induction of B7-H1 expression in the corneal epithelium and up-regulation of T-cell surface PD-1. B7-H1 is constitutively expressed in the endothelial cells and stromal cells of the normal cornea but not in the epithelial cells. PD-1 expression on the surface of
T cells is up-regulated by contact with corneal cells. In the presence of IFN- $\gamma$, B7-H1 expression on epithelial cells is induced, and T-cell apoptosis mediated by B7-H1 and PD-1 is induced in each of the epithelium, stroma, and endothelium of the cornea 
phenomena will contribute to the development of new therapeutic approaches to tissue transplantation and autoimmune disease of not only the eye but also of other organs.

Acknowledgments The author dedicates this review to the memory of her mentor, Dr. J. Wayne Streilein. Dr. Streilein had made an enormous contribution to the understanding of immune privilege.

Disclosures The author does not have any commercial relationship to disclose.

\section{References}

1. Streilein JW. Ocular immune privilege: therapeutic opportunities from an experiment of nature. Nat Rev Immuno. 2003;3:879-89.

2. Niederkorn JY. See no evil, hear no evil, do no evil: the lessons of immune privilege. Nat Rev Immuno. 2006;7:354-9.

3. Hori J, Streilein JW. Survival in high-risk eyes of epitheliumdeprived orthotopic corneal allografts reconstituted in vitro with syngeneic epithelium. Invest Ophthalmol Vis Sci. 2003;44:658-64.

4. Medawar PB. Immunity to homologous grafted skin. III. The fate of skin homografts transplanted to the brain, to subcutaneous tissue, and to the anterior chamber of the eye. Br J Exp Pathol. 1948;29:58-69.

5. Streilein JW, Toews GB, Bergstresser PR. Corneal allografts fail to express Ia antigens. Nature 1979;282:326-7.

6. Wang HM, Kaplan HJ, Chan WC, et al. The distribution and ontogeny of MHC antigens in murine ocular tissue. Invest Ophthalmol Vis Sci. 1987;28:1383-9.

7. Sonoda Y, Streilein JW. Orthothopic corneal transplantation in mice - evidence that the immunogeneic rules of rejection do not apply. Transplantation 1992;54:694-704.

8. Streilein JW, Arancibia CC, Osawa H. In adequate HLA matching in keratoplasty. In: Sundmacher R, editor. Developmental ophthalmology. vol 36. Basel: Karger AG; 2003. p. 74-88.

9. Hamrah P, Zhang Q, Liu Y, et al. Novel characterization of MHC class II-negative population of resident corneal Langerhans celltype dendritic cells. Invest Ophthalmol Vis Sci. 2002;43:639-46.

10. Sonoda Y, Streilein JW. Impaired cell-mediated immunity in mice bearing healthy orthotopic corneal allograft. J Immunol. 1993; 150:1727-34.

11. Hara Y, Caspi RR, Wiggert B, Chan CC, Streilein JW. Use of ACAID to suppress interphotoreceptor retinoid binding proteininduced experimental autoimmune uveitis. Curr Eye Res. 1992;11 (Suppl):97-100.

12. Rochat C, Polla BS, Herbort CP. Immunological profiles in patients with acute retinal necrosis. Graefes Arch Clin Exp Ophthalmol. 1996;234:547-52.

13. Kezuka T, Sakai J, Usui N, Streilein JW, Usui M. Evidence for antigen-specific immune deviation in patients with acute retinal necrosis. Arch Ophthalmol. 2001;119:1044-9.

14. Kezuka T. Immune deviation and ocular infections with varicella zoster virus. Ocul Immunol Inflamm. 2004;12:17-24.

15. Willbanks GA, Mammolenti MM, Streilein JW. Studies on the induction of anterior chamber-associated immune deviation (ACAID). III. Induction of ACAID depends upon intraocular transforming growth factor- $\beta 2$. Eur J Immunol. 1992;22:165-173.

16. Sheibani N, Sorenson CM, Cornelius LA, et al. Thrombospondid1 , a natural binhibitor of angiogenesis, is present in vitreous and aqueous humor and is modulated by hyperglycemia. Biochem Biophys Res Commun. 2000;267:257-68.

17. Willbanks GA, Streilein JW. Studies on the induction of anterior chamber-associated immune deviation (ACAID). 1 Evidence that an antigen-specific, ACAID-inducing, cell-associated signal exists in the peripheral blood. J Immunol. 1991;146:2610-7.

18. Wang Y, et al. Blood mononuclear cells induce regulatory NKT thymocytes in anterior chamber-associated immune deviation. J Leukoc Biol. 2001;69:741-6.

19. Faunce DE, Stein-Streilein J, et al. MIP-2 recruits NKT cells on the spleen during tolerance induction. J Immunol. 2001;166:31321.

20. Sonoda KH, Stein-Streilein J, et al. CD1d-reactive natural killer T cells are required for development of systemic tolerance through and immune-privileged site. J Exp Med. 1999;190:1215-26.

21. Faunce DE, Stein-Streilein J, et al. NKT cells-derived RANTES recruits APCs and CD8 $+\mathrm{T}$ cells to the spleen during the generation of regulatory $\mathrm{T}$ cells in tolerance. J Immunol. 2002; 169:31-8.

22. Sonoda KH, Stein-Streilein J, et al. NKT cell-derived IL-10 is essential for the differentiation of antigen-specific $\mathrm{T}$ regulatory cells in systemic tolerance. J Immunol. 2001;166:42-50.

23. D’Orazio TJ, Niederkorn JY. Splenic B cells are required for tolerogenic antigen presentation in the induction of anterior chamber-associated immune deviation (ACAID). Immunology 1998;95:47-55.

24. Skelsey ME, et al. Splenic B cells act as antigen presenting cells for the induction of anterior chamber-associated immune deviation. Invest Ophthalmol Vis Sci. 2003;44:5242-51.

25. Skelsey ME, et al. CD25+, interleukin-10-producing CD4+ T cells are required for suppressor cell production and immune privilege in the anterior chamber of the eye. Immunology 2003;110:18-29.

26. Nakamura T, Stein-Streilein J, et al. CD4+ NKT cells, but not conventional CD4 + T cells, are required to generate efferent CD8+ $\mathrm{T}$ regulatory cells following antigen inoculation in an immune privileged site. J Immunol. 2003;171:1266-71.

27. Skelsey ME, et al. gdT cells are needed for ocular immune privilege and corneal graft survival. J Immunol. 2001;166:4327-33.

28. Niederkorn JY, Mellon J. Anterior chamber-associated immune deviation promotes corneal allograft survival. Invest Ophthalmol Vis Sci. 1996;37:2700-7.

29. Sonoda KH, Stein-Streilein J, et al. Long-term survival of corneal allografts is dependent on intact CD1d-reactive NKT cells. J Immunol. 2002;168:2028-34.

30. Namba K, Kitaichi N, Nishida $\mathrm{T}$, et al. Identification of amelanocyte stimulating hormone and transforming growth factorß2. J Leukoc Biol. 2002;72:946-52.

31. Taylor AW, Yee DG, Streilein JW. Suppression of nitric oxide generated by inflammatory macrophages by calcitonin generelated peptide in aqueous humor. Invest Ophthalmol Vis Sci. 1998;39:1372-8.

32. Apte RS, Sinha D, Mayhew E, et al. Role of macrophage migration inhibitory factor in inhibition NK cell activity. J Immunol. 1998;160:5693-6.

33. Kennedy MC, Rosenbaum JT, Brown J. Novel production of interleukin-1 receptor antagonist peptides in normal human cornea. J Clin Invest. 1995;95:82-8.

34. Shon JH, Kaplan HJ, Suk HJ, et al. Complement regulatory activity of normal human intraocular fluid is mediated by MCP, DAF, and CD59. Invest Ophthalmol Vis Sci. 2000;41:4195-4202.

35. Griffith TS, Brunner T, Fletcher SM, et al. FAS ligand-induced apoptosis as a mechanism of immune privilege. Science 1995;270:1189-92. 
36. Hori J, Joyce NC, Streilein JW. Epithelium-deficient corneal allografts display immune privilege beneath the kidney capsule. Invest Ophthalmol Vis Sci. 2000;41:443-52.

37. Hori J, Joyce NC, Streilein JW. Immune privilege and immunogenicity reside among layers of the mouse cornea. Invest Ophthalmol Vis Sci. 2000;41:3032-42.

38. Sugita S, Streilein JW. Iris pigment epithelium expressing CD86 (B7-2) directly suppresses T cell activation in vitro via binding to cytotoxic $\mathrm{T}$ lymphocyte-associated antigen 4. J Exp Med. 2003;198:161-71.

39. Hori J, Wang MC, Miyashita M, et al. B7-H1-induced apoptosis as a mechanism of immune privilege of corneal allografts. J Immunol. 2006;177:5928-35.
40. Dong H, Zhu G, Tamada K, et al. B7-H1, a third member of the B7 family, co-stimulates T-cell proliferation and interleukin-10 secretion. Nat Med. 1999;5:1365-9.

41. Dong H, Strome SE, Salomao DR, et al. Tumor-associated B7-H1 promotes T-cell apoptosis: a potential mechanism of immune evasion. Nat Med. 2002;8:793-800.

42. Dong H, Zhu G, Koji T, et al. B7-H1 determines accumulation and deletion of intrahepatic $\mathrm{CD} 8+\mathrm{T}$ lymphocytes. Immunity 2004;20:327-36.

43. Chapoval AL, Ni J, Lau JS, et al. B7-H3: a costimulatory molecule for T cell activation and IFN- $\gamma$ production. Nat Immunol. 2001;2:269-74.

44. Taniguchi H, Hori J, Wang MC, et al. Role of B7-H3 on immune privilege of corneal allografts. Proc Jpn Soc Immunol. 2007;37:115. 